\section{MS34-P10 Multitopic precursors for oxide materials' synthesis}

Alba Finelli ${ }^{1}$, Aurélien Crochet $^{1}$, Katharina M. Fromm ${ }^{1}$

1. University of Fribourg, Switzerland

email: alba.finelli@unifr.ch

Research interest in mixed metal oxide is increasing in material science. They have multiple applications, such as batteries, ceramics, pigments, high-Tc superconductors or transparent conductors. However, the two main challenges for the synthesis of such compounds are the lack of control on the ratio of the different metal components and the extreme conditions (up to $900{ }^{\circ} \mathrm{C}$ ) that many of these oxides require during the synthesis. To overcome these kinds of issues we propose a new strategy for the synthesis of mixed metal complexes, a "multitopic ligand approach".The aim is to design specific ligands with selective coordination sites to bind different metal ions. Due to the metal ion preorganization in the precursor thus formed, the stoichiometry of the final oxide material can be controlled and the extreme synthesis conditions diminished (pressure or temperature). These new mixed metal complexes will be finally combusted to oxide materials with possible new features and ideally at the nanoscale.

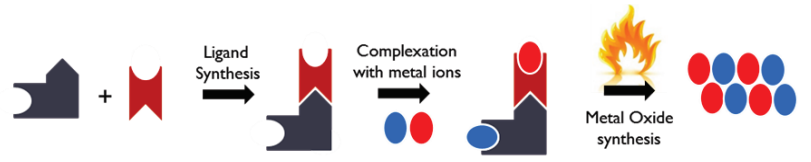

Figure 1. Multitopic ligand approach

Keywords: Multitopic ligand, coordination chemistry, oxides
MS34-P11 Co-crystals of vanillic acid with methylated xanthines

Ayesha Jacobs ${ }^{1}$, Francoise M. Amombo $\mathrm{Noa}^{1}$

1. Cape Peninsula University of Technology

email: jacobsa@cput.ac.za

Vanillic acid (VA) forms co-crystals with caffeine (CAF) and theophylline (THP) of the form VA•2CAF and 2VA $\cdot$ THP respectively. VA also forms a co-crystal hydrate with theobromine (THB), VA•THB $2 \mathbf{H}_{2} \mathbf{O}$. The structures of the co-crystals were determined. For VA-2CAF, each VA molecule is hydrogen bonded to two CAF molecules to form a unit. The units are linked via weak $\mathrm{CH} \cdots \cdot \mathrm{N}$ and $\mathrm{CH} \cdots \mathrm{O}$ interactions. The hydrogen bond network observed for $\mathbf{2 V A} \cdot \mathbf{T H P}$ is more extensive due to the additional N-H donor group of the THP. The hydrogen bonded ring networks pack to form layers with $\pi \cdots \pi$ interactions between the layers. The packing for $\mathbf{V A} \cdot \mathbf{T H B} \cdot 2 \mathrm{H}_{2} \mathrm{O}$ also presents with hydrogen bonded layers with the water molecules acting as bridges linking the VA-THB dimers. VA-2CAF was successfully prepared via the slurry method and via neat grinding. $\mathbf{V A} \cdot \mathbf{T H B} \cdot 2 \mathrm{H}_{2} \mathrm{O}$ could not be prepared via the previous two methods, however liquid assisted grinding was successful. Grinding experiments utilising 2: 1 mixtures of VA: THP resulted in the dihydrate, $\mathbf{2 V A} \cdot \mathbf{T H P} \cdot \mathbf{2} \mathbf{H}_{2} \mathbf{O}$.

Keywords: vanillic acid, caffeine, theobromine, theophylline, co-crystals, grinding 